\title{
Caracterización de la harina de cáscara de chocho (Lupinus mutabilis Sweet) para aplicaciones agroindustriales y medicinales
}

DOI: https://doi.org/10.33262/ap.v3i2.2.55

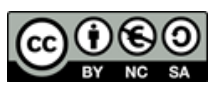

\section{Characterization of lupine shell flour (Lupinus mutabilis Sweet) for agroindustrial and medicinal applications}

\section{Sonia Lourdes Rodas Espinoza. ${ }^{1}$, Jessica Alexandra Marcatoma Tixi. ${ }^{2}$, Angela del Rocío Calderón. ${ }^{3} \&$ Lourdes Flora Cuadrado Merino. ${ }^{4}$}

\begin{abstract}
.
Introduction: Choco husk is an agro-industrial waste generated by premises that are dedicated to the sale and marketing of this legume, despite having excellent nutritional properties, they have not been used and continue to be a waste in the transformation line. Objetive: Characterize lupine shell flour (Lupinus mutabilis Sweet) through bromatological, macro and micronutrient and microbiological analyzes. Methodology: The pre-experimental study was developed in the Laboratories of the Agroindustrial Engineering career of the Faculty of Engineering of the National University of Chimborazo and of the State University of Bolívar; As experimental material, $4 \mathrm{~kg}$ of debittered lupine shells were used INIAP 450 for the subsequent elaboration of the flour for the bromatological and microbiological characterization of the product. Results: In the bromatological analysis, high percentages of fiber (73.4) and protein (11.3) were

\footnotetext{
${ }^{1}$ Universidad Nacional de Chimborazo, Facultad de Ingeniería, Riobamba, Ecuador, srodas@unach.edu.ec ${ }^{2}$ Universidad Nacional de Chimborazo, Facultad de Ingeniería, Riobamba, Ecuador, jessica.marcatoma@unach.edu.ec

${ }^{3}$ Universidad Nacional de Chimborazo, Facultad de Ingeniería, Riobamba, Ecuador, hmullo@espoch.edu.ec

${ }^{4}$ Escuela Superior Politécnica de Chimborazo, Facultad de Ciencias, Riobamba, Ecuador, nperez@espoch.edu.ec
} 
observed compared to the NTE INEN 2 390: 2004 2005-9 norm, in terms of macro and micronutrients, the calcium values $(4896.15 \mathrm{mg} / \mathrm{kg}$ ) stood out, Potassium $(1068.5 \mathrm{mg} /$ $\mathrm{kg}$ ) and iron (58.5 mg / kg) compared to the NTE-INEN-616: 2006-01 standard, the presence of molds and yeasts, Mesophilic Aerobes and Escherichia coli was controlled by the NTE-INEN standard- 616: 2006-01; which guarantees product safety. Conclusions: With the properties found, it is expected that the flour will be used as an additive in the elaboration of functional and pharmacological foods.

Keywords: Chocho, Flour, Fiber, Calcium

\section{Resumen.}

Introducción: La cáscara de chocho es un residuo agroindustrial generado por locales que se dedican al expendio y comercialización de esta leguminosa, a pesar de contar con excelentes propiedades nutricionales no han sido utilizadas y continúan siendo un desecho en la línea de transformación. Objetivo: Caracterizar la harina de cáscara de chocho (Lupinus mutabilis Sweet) mediante análisis bromatológicos, de macro y micronutrientes y microbiológicos. Metodología: El estudio preexperimental se desarrolló en los Laboratorios de la carrera de Ingeniería Agroindustrial de la Facultad de Ingeniería de la Universidad Nacional de Chimborazo y de la Universidad Estatal de Bolívar; como material experimental se utilizó $4 \mathrm{~kg}$ de cáscaras de chocho desamargado INIAP 450 para la elaboración de la harina para la posterior caracterización bromatológica y microbiológica del producto. Resultados: En el análisis bromatológico se observó porcentajes elevados de fibra (73.4) y proteína (11.3) comparados con la norma NTE INEN 2 390:2004 2005-9, en cuanto a macro y micronutrientes sobresalieron los valores de calcio $(4896.15 \mathrm{mg} / \mathrm{kg})$, potasio $(1068.5 \mathrm{mg} / \mathrm{kg})$ y hierro $(58.5 \mathrm{mg} / \mathrm{kg})$ comparados con la norma NTE-INEN-616:2006-01, la presencia de mohos y levaduras, Aerobios mesófilos y Escherichia coli estuvo controlada por la norma NTE-INEN616:2006-01; lo que garantiza inocuidad del producto. Conclusiones: Con las propiedades halladas se espera que la harina sea utilizada como aditivo en la elaboración de alimentos funcionales y farmacológicos

Palabras claves: Chocho, Harina, Fibra, Calcio

\section{Introducción.}

Los granos de chocho (Lupinus mutabilis Sweet), han sido desde tiempos inmemoriales parte esencial en la alimentación de nuestros pueblos; pero el desarrollo de las sociedades ha producido una disminución de su consumo. El perfil nutricional de esta leguminosa y las cáscaras alojadas en el grano, son una fuente rica de compuestos bioactivos como fibra dietética, minerales, vitaminas, así como también a la presencia de compuestos fitoquímicos. (Falconi, 2012). 
Varios estudios in vitro e in vivo, a nivel preclínico y clínico, han demostrado que el consumo de granos enteros y/o de productos elaborados a partir de los componentes bioactivos del chocho, son capaces de producir efectos preventivos y/o terapéuticos sobre diferentes enfermedades crónicas no transmisibles. (Cuadrado, 2019) rescatando la importancia de la fibra dietética, a la cual se le atribuye propiedades diversas como: ser un regulador intestinal al actuar como laxante; representa un factor preventivo del cáncer de colon y ayuda favorablemente aquellas patologías relacionadas con el Síndrome Metabólico. (Cuadrado, 2019; Sanaguano et al., 2017; Lattimer \& Haub, 2010; Falconi, 2012; Horton, 2014.)

Sin embargo, a pesar de la revalorización y al reconocimiento que hoy se le da a este grano andino por todos sus componentes nutricionales (Quelal, 2019); se puede evidenciar que en la ciudad de Riobamba, en los sitios donde se elaboran y comercializan los tradicionales ceviches de chocho, continúan retirando la piel o tegumento de estos granos "sólo por agradar al paladar de los comensales"; dejando a un lado la calidad nutracéutica de éstos. (Ibarra \& Yépez , 2017). Además, éstos residuales terminan siendo una fuente de contaminación ambiental que promueven la propagación de plagas, malos olores, contaminación del suelo, entre otros. (Serna Cock \& Torres León, 2015)

Bajo esta perspectiva, el objetivo de este trabajo de investigación fue, caracterizar la composición bromatológica de las cáscaras del grano de chocho, para su posterior uso en el desarrollo de nuevos productos funcionales.

\section{Metodologia.}

En la investigación se aplicó un diseño pre experimental donde no se manipulan tratamiento ni grupos de control (Baray, 2006), al tratarse de la caracterización bromatológica de los macro y micronutrientes; así como también un análisis microbiológico de un producto como lo es la harina de las cáscaras de chocho. El estudio se desarrolló en los Laboratorios de la carrera de Ingeniería Agroindustrial de la Facultad de Ingeniería de la Universidad Nacional de Chimborazo y de la Universidad Estatal de Bolívar; como material experimental se utilizó 4 kg de cáscaras de chocho desamargado INIAP 450.

Los análisis bromatológicos que se llevaron a cabo fueron el de humedad, acidez (NTEINEN-616:2006-01), proteína, cenizas, grasa, fibra (NTE INEN 2 390:2004 2005-9), las normas para el estudio de macronutrientes fue CODEX STAN 152-1985 y NTE-INEN616:2006-01 en potasio, calcio, hierro. Se realizó el recuento de mohos y levaduras, Aerobios mesófilos y Escherichia coli conforme la norma NTE-INEN-616:2006-01.

\section{Elaboración de la harina de cáscara de chocho}

Luego de la recepción de $4 \mathrm{~kg}$ de cáscara de chocho obtenidos de sitios de elaboración y expendio de ceviches de chochos de la ciudad de Riobamba, se procedió a la selección de la materia prima con la finalidad de desechar las cáscaras que se encontraban en mal estado y las impurezas adheridas, el producto seleccionado fue sometido a lavado con 8 
L de agua destilada con el fin de evitar una contaminación microbiana, para disminuir el contenido de humedad de la cáscara, se utilizó un secador de bandejas a una temperatura de $60^{\circ} \mathrm{C}$ durante 5 horas, las cáscaras deshidratadas pasaron por un proceso de molienda en un molino de mano, seguido del tamizaje de la harina en tamices \# 10, 20, 40, 60, 80 y $100 \mu \mathrm{m}$ de acuerdo a la norma NTE INEN 6162015 - 01. Al término del proceso se empacó la harina en fundas herméticas de polietileno y se almacenó en lugares frescos, a temperatura ambiente $\left(15{ }^{\circ} \mathrm{C}\right) \mathrm{y}$ humedad relativa $(76 \%)$.

\section{Resultados.}

\section{Análisis Bromatológico de la harina de cáscara de chocho}

En la tabla 1, se muestran los valores en base seca obtenidos del análisis bromatológico de la harina de cáscara de chocho (Lupinus mutabilis Sweet)

\begin{tabular}{cccc}
\hline Determinación & $\%$ & $\begin{array}{c}\text { Valores de referencia } \\
\text { según la norma NTE- } \\
\text { INEN-616:2006-01 }\end{array}$ & Método de ensayo \\
\hline Humedad & 6,7 & $14.5^{*}$ & NTE INEN 518 \\
Cenizas & 2,7 & $1,9-3,0$ & AOAC 942.05 \\
Proteína & 11,3 & $50-52$ & AOAC 955.04 \\
Grasa & 8,5 & $19-24$ & AOAC 920.85 \\
Fibra & 73,4 & $7-9$ & AOAC 962.09 \\
Acidez & 0,03 & $0.1^{*}$ & NTE INEN 521 \\
\hline
\end{tabular}

*Valor máximo del método de ensayo.

-Valor mínimo del método de ensayo.

Tabla 1.Análisis proximal de la cáscara de chocho Fuente: Elaboración propia

Observándose que, en cuanto al contenido de humedad, es inferior al referido en la norma NTE-INEN-616:2006-01, lo que garantizaría que no se desarrollen microrganismos durante el almacenamiento del producto. (INEN, 2006), en cuanto al porcentaje de cenizas, el valor obtenido es superior al contenido del grano entero desamargado $(2,54 \%)$ valor reportado por Allauca (2005). Esta diferencia se debe a que el calcio se encuentra principalmente en la cáscara. (Cuadrado, 2019). El contenido proteico de las cáscaras de chocho es inferior al grano entero cuyo valor oscila entre el $41 \%$ y $54 \%$ en base seca. Sin embargo, es importante indicar que el contenido proteico de la cáscara de chocho es superior a los cereales que se consumen cotidianamente como son el arroz (6,77\%), maíz $(10,2 \%)$, trigo $(10,4 \%)$. (Cuadrado, 2019).

Es relevante indicar que la calidad proteica del chocho no sólo está definida por su cantidad sino también por su valor biológico, el cual está determinado por a) la alta digestibilidad cuyo valor supera el $90 \%$ siendo similar al de la caseína (91.9\%), pero superior a los valores de la mayoría de leguminosas como arvejas y frijoles las cuales están por debajo del $80 \%$; y b) por el perfil aminoácidico, similar a la soja, cuyos valores son muy cercanos a los requerimientos establecido por la FAO. (Cuadrado, 2019). 
El contenido de lípidos en la harina de cáscaras de chochos es inferior al grano entero $(21,22 \%)$, sin embargo, es superior a los valores encontrados en algunas leguminosas como frejol (7\%), habas (3\%), lentejas $(1,8 \%)$, así como también a la de algunos cereales como el arroz (2\%), maíz (7,5\%), trigo $(3,4 \%)$. (Cuadrado, 2019).

Estudios realizados por Chirinos-Arias (2015), muestran que en los granos enteros de chocho hay una mayor concentración de ácidos grasos poliinsaturados: Oleico $(\omega 9)$, Linoleico $(\omega 6)$ y Linolénico $(\omega 3)$ que representan el $51,13 \% ; 37,1 \%$ y $2,9 \%$ del total respectivamente; en cambio, los ácidos grasos saturados se hallan en menor proporción palmítico $(13,4 \%)$ y esteárico constituyen $(5,7 \%)$. Los ácidos grasos poliinsaturados son esenciales para nuestro organismo, cumplen varias funciones como: proteger al sistema cardiovascular, tienen efecto antitumoral, son precursores para la formación de prostaglandinas, hormonas importantes en el control de la tensión arterial y funcionamiento de las plaquetas, ayudan al correcto funcionamiento del cerebro y la estimulación de las conexiones neuronales, protegen la retina, tiene poder antiinflamatorio y anticoagulante. Además, las semillas de chocho contienen fitoesteroles de los cuales el más destacado es el $\beta$-sitosterol por su efecto hipolipidémico. (Cuadrado, 2019)

En la harina de las cáscaras de chocho es evidente el alto contenido de fibra dietética, cuyo valor supera al reportado en el grano entero desamargado de chocho (14\%), así como también a la mayoría de leguminosas y cereales. (Cuadrado, 2019). Estudios realizados por Ortega et al (2010) reportan que la fibra del Lupinus mutabilis procedente de Colombia es rica en celulosa, hemicelulosa y lignina (37\%, $7 \%$ y $30 \%$ respectivamente), en proporción mayor en la cáscara que el cotiledón considerándose de elevado valor nutritivo.

El chocho posee dos tipos de fibra: la fibra soluble (similar a la avena) y la fibra insoluble (como la del salvado de trigo), cada una presenta funciones diferentes. La fibra insoluble, posee una escasa viscosidad y una baja fermentabilidad colónica; ésta juega un papel importante en la fisiología humana por sus propiedades funcionales como son la capacidad de retención de agua, de intercambio catiónico y formación de geles, es capaz de retener agua en la matriz estructural por lo cual aumenta la velocidad del tránsito intestinal produciendo un efecto laxante, que limpia los intestinos. Ayuda al tratamiento de la diverticulosis, así como también previene cáncer de colon. (Jácome, 2017), (Cuadrado, 2019), el consumo frecuente de productos ricos en fibra según (Mayo Clinic, 2019) reduce el riesgo de presentar diabetes tipo 2; su consumo frecuente garantiza la ausencia de enfermedades cardiovasculares y el desarrollo de cualquier tipo de cáncer.

En cambio, la fibra soluble al llegar al intestino grueso se fermenta produciendo ácidos grasos de cadena corta, los cuales promueven el crecimiento de bacterias intestinales benéficas como las bifidobacterias; reducen el crecimiento de bacterias intestinales nocivas como Clostridium (C. ramosum, C. spiroforme, y C. Cochleatum); incrementa la saciedad, lo cual a su vez reduce la ingesta calórica y con ello ayudaría en la reducción 
de los niveles glicémicos y lipídicos en sangre; así como también favorecerá en el control del peso corporal. (Cuadrado, 2019).

Además, la fibra soluble, es muy utilizada en la industria alimenticia como aditivo para la formación de geles, como espesantes o estabilizantes. Estudios realizados por Huygue (1997) reportan que la fibra del lupino presenta elevada capacidad de retención de agua ( $7.1 \mathrm{~g} \mathrm{H}_{2} \mathrm{O} / \mathrm{g}$ lupino) lo cual hace útil a la harina integral de lupino como componente en productos dietarios, ya que se conoce que la fibra ayuda en el metabolismo de la glucosa, transporta micronutrientes y disminuye la absorción de colesterol. (Jácome, 2017)

En la tabla 2 y 3 se muestra el análisis de macro y micronutrientes de la harina de las cáscaras de chocho

\begin{tabular}{cccc}
\hline Determinación & $\mathbf{m g} / \mathbf{k g}$ & $\begin{array}{c}\text { Valores de Referencia } \\
\text { según la norma } \\
\mathbf{m g} / \mathbf{k g}\end{array}$ & Norma \\
\hline Potasio & 1068,5 & $300^{*}$ & CODEX STAN 152-1985 \\
Calcio & 4896,15 & $300^{*}$ & CODEX STAN 152-1985 \\
Fósforo & 19,61 & & CODEX STAN 152-1985 \\
\hline *Valor máximo de la norma & &
\end{tabular}

*Valor máximo de la norma.

-Valor mínimo de la norma.

Tabla 2.Análisis de macronutrientes la cáscara de chocho Fuente: Elaboración propia

$\frac{\mathrm{mg} / \mathrm{g}}{58,5}$
Tabla 3.Análisis de micronutrientes la cáscara de chocho
Fuente: Elaboración propia

Los resultados permitieron afirmar que el calcio es el mineral más representativo en el chocho y éste se encuentra en mayor concentración en el tegumento que en los cotiledones (1,07 mg/g -1,53 mg/g); en cambio el resto de minerales (fósforo, magnesio, hierro y potasio) se hallan principalmente en el grano, demostrando que la relación de calcio frente al resto de minerales se altera tras el descascarado del grano. (Cuadrado, 2019).

Los minerales son considerados como nutrientes indispensables, pues el organismo humano no los puede sintetizar; y éstos desempeñan funciones fisiológicas elementales. A los minerales se los ha dividido en dos grupos: los Macroelementos ( $\mathrm{Ca}, \mathrm{P}, \mathrm{Mg}, \mathrm{Cl}, \mathrm{S}$ ) que son los que el organismo necesita en mayor cantidad (100 mg/día) y los miroelementos ( $\mathrm{Fe}, \mathrm{Cu}, \mathrm{F}, \mathrm{Mn}, \mathrm{Zn}, \mathrm{Cr}, \mathrm{Se}, \mathrm{Mo}, \mathrm{Co}$ ) que se necesitan en menor cantidad, unos pocos mg/día. Muchos de ellos actúan como cofactores enzimáticos, otros son de suma importancia para controlar la presión osmótica de fluidos celulares y el pH, o bien forman parte constitutiva de algunas macromoléculas. (Carrión, 2006) 
La presencia de estos macro y micronutrientes en un producto alimenticio aporta al organismo beneficios importantes, según (Dergal, 2006) así por ejemplo algunos estudios comunicaron que la ingesta alta de potasio protege contra la hipertensión y otros riesgos cardiovasculares (Velásquez, 2012); asimismo (Bowman, 2003), en un metaanálisis observó que suplementos y productos ricos en potasio reducen significativamente las presiones sistólica $(-5,9 \mathrm{~mm} \mathrm{Hg})$ y diastólica $(-3,4 \mathrm{~mm} \mathrm{Hg})$.

El calcio por su parte aporta a la mineralización de huesos y dientes, la regulación de las funciones celulares en prácticamente todos los tejidos corporales según (Victoria, 2016), en torno a las características del producto se espera que los consumidores principales sean los niños ya que la ingesta de calcio contribuye en los primeros años de vida al crecimiento en longitud de los huesos largos y las mujeres especialmente cuando pasan el proceso de menopausia en donde se produce la mayor pérdida ósea de calcio, también evita la mayor incidencia de fracturas óseas de estrés, más frecuentes en esta etapa (Victoria, 2016)

Otro beneficio que reciben los niños y mujeres es el aporte de hierro que almacena la harina, sobre todo cuando se trata de combatir anemias en estos grupos de la población, contribuyendo al incremento del apetito y a mejorar la capacidad de aprendizaje y crecimiento de los menores (Latham, 2002), en el caso de las consumidoras femeninas el producto sería esencial para el cumplimiento de planes nutricionales y dietarios (Lezcano, 2010)

Los resultados del análisis microbiológico de la harina de las cáscaras de chocho (ver tabla 4), muestran que ésta contiene una baja carga microbiológica con respecto a los valores de referencia dadas en las normas INEN (NTE INEN: 1529-10; 1529-5 y 15298).

\begin{tabular}{cccc}
\hline Determinación & UFC/g & $\begin{array}{c}\text { Valores de referencia } \\
\text { según las Normas } \\
\text { INEN } \\
\text { UFC/g }\end{array}$ & Método de ensayo \\
\hline Mohos y Levaduras & $1.7 \times 10^{2}$ & $5 \times 10^{2}$ & NTE INEN 1 529 - 10 \\
Aerobios mesófilos & $1.1 \times 10^{3}$ & $1 \times 10^{5}$ & NTE INEN 1 529 - 5 \\
Escherichia coli & Ausencia & Ausencia & NTE INEN 1 529 - 8 \\
\hline
\end{tabular}

Tabla 4.Análisis microbiológico de la harina de la cáscara de chocho Fuente: Elaboración propia

Es importante indicar que las condiciones de almacenamiento: temperatura $\left(15^{\circ} \mathrm{C}\right)$, humedad relativa (60\%), así como también el contenido de humedad en la harina $(6,7 \%)$ son factores críticos en el control del desarrollo de microorganismos. Por lo tanto, al tener controlados estos parámetros se garantizó la inocuidad de este producto, considerándose apto para el consumo humano. 


\section{Conclusiones.}

- La harina de cáscara de chocho almacena un gran valor nutricional debido a su alto contenido de fibra que no puede ser sintetizado por el organismo y que lo requiere en la ingesta de su dieta diaria, el uso del producto en suplementos alimenticios y farmacológicos aportará de forma significativa no solo a la mejora de salud de los comensales sino también al saneamiento ambiental a través del uso y aplicación de tecnologías limpias, ya que hasta el momento las cáscaras de chocho siguen siendo un desecho no utilizado.

- En la industria de alimentos la fibra dietética puede ser utilizada como aditivo para la elaboración de alimentos funcionales como yogurt, kumis, galletas, los cuales aportarán beneficios para la prevención y/o tratamiento de enfermedades crónico-degenerativas relacionadas con el Síndrome Metabólico, además promoverá efectos fisiológicos, actuando como laxante a nivel del aparato digestivo y reduciendo los niveles de colesterol y glucosa a nivel hematológico.

- Se espera que el uso del producto se direccione especialmente a pacientes diabéticos, quienes requieren dosis elevadas de fibra como parte de su patrón alimentario.

\section{Referencias bibliográficas.}

Baray, H. Á. (2006). Introdución a la metodología de la investigación (Edición electrónica ed.). México. Obtenido de www.eumed.net/libros2006c/203/

Bowman, B. (2003). Conocimientos actuales sobre nutrición. Revista del Instituto de Medicina Tropical de São Paulo, 873.

Carrión, J. (2006). Reutilización del efluente del desamargado de chocho (Lupinus mutabilis Sweet). (Ingeniería en Alimentos ). Universidad Técnuica de Ambato, Ambato.

Obtenido

de https://books.google.com.ec/books?id=9nozAQAAMAAJ\&pg=RA1PA20\&lpg=RA1-

PA20\&dq=el+contenido+de+minerales+se+encuentra+en+mayor+cantidad+en+ los+cotiledones+o+en+la+c\%C3\%A1scara\&source $=$ bl\&ots $=S P q 0 z a M q W Q \& s i$ $\mathrm{g}=$ ACfU3U0QGTkJ0r7-wz2wnDKV8PyaMLN_eg\&hl=es-419\&sa=X\&v

Chirinos-Arias, M. C. (julio de 2015). Andean Lupin (Lupinus mutabilis Sweet) a plant with nutraceutical and medicinal potentia. Revista BIO CIENCIAS, 3((3)), 163172. https://www.researchgate.net/publication/281034234_Andean_Lupin_Lupinus_ mutabilis_Sweet_a_plant_with_nutraceutical_and_medicinal_potential

Cuadrado, L. (2019). Lupinus mutabilis Sweet, como tratamiento complementario en pacientes con diabetes mellitus tipo II, del club de diabéticos del Hospital General 
Docente de la ciudad de Riobamba durante el periodo septiembre 2017 diciembre 2018. (Tesis de Maestría). Universidad Tecnica Particular de Loja, Riobamba.

Dergal, S. B. (2006). Química de los alimentos (Cuarta Edición ed.). México: Pearson educación.

Falconi, C. (2012). Lupinus mutabilis in Ecuador with special emphasis on anthracnose resistance. Tesis de Doctorado. Wageningen University, Wageningen, Países Bajos. doi:10.1079/BJN20031088

FAO. (11 de Noviembre de 2016). El gobierno del ecuador y la FAO impulsan la investigacion cientifica del chocho. Obtenido de http://www.fao.org/ecuador/noticias/detail-events/en/c/453499/

Hall, R., Baxter, A., Johnson, S., \& Ball, M. (2005). Lupin kernel fibre-enriched foods beneficially modify serum lipids in men. European Journal of Clinical Nutrition, 59(3), 325-33. doi:10.1038 / sj.ejcn.1602077

Horton, D. (2014). Investigación Colaborativa de Granos Andinos en Ecuador. Quito.

Ibarra , L., \& Yépez , G. (2017). Análisis Gastronómico del Ceviche de Chocho en la Ciudad de Riobamba. (Tesis de Lincenciatura). Universidad de Gyuayaqui, Guayaquil. Obtenido de http://repositorio.ug.edu.ec/bitstream/redug/20761/1/TESIS\%20Gs.\%20205\%20 -\%20Anali\%20Gastron\%20del\%20Ceviche\%20de\%20Chocho.pdf

INEN. (2006). NTE INEN 0616: Harina de trigo. Requisitos. Obtenido de NORMATÉCNICAECUATORIANANTE INEN616:200: https://studylib.es/doc/5531663/nte-inen-0616--harina-de-trigo.-requisitos

Jácome, X. (2017). Efecto del proceso de desamargado y fermentado en el contenido de fibra dietética y. (Licenciatura en Ciencias Químicas con Mención en Química Analítica). PONTIFICIA UNIVERSIDAD CATÓLICA DEL ECUADOR, Quito. Obtenido de http://repositorio.puce.edu.ec/bitstream/handle/22000/14210/TESISFINAL $\% 20 \% 282 \% 29$.pdf? sequence=1\&isAllowed=y

Latham, M. (2002). Nutrición humana en el mundo en desarrollo. En M. Latham, Nutrición humana en el mundo en desarrollo (págs. 160-165).

Lattimer, J. M., \& Haub, M. D. (5 de Diciembre de 2010). Effects of Dietary Fiber and Its Components on Metabolic Health. Nutrients, 2((12)), pp. 1266-1289. doi:10.3390/nu2121266

Lezcano, E. (2010). Análisis de producto: cereales para el desayuno. Argentina: Alimentos argentinos-MinAgri. 
Mayo Clinic. (20 de junio de 2019). Nutrición y comida saludable. Obtenido de Nutrición y comida saludable: https://www.mayoclinic.org/es-es/healthy-lifestyle/nutritionand-healthy-eating/in-depth/fiber/art-20043983

Ortega, E., Rodríguez, A., David, A., \& Zamora-Burbano, Á. (2010). Caracterización de semillas de lupino (Lupinus mutabilis) sembrado en los Andes de Colombia. Acta Agronómica, $\quad 59((19))$ Obtenido de http://www.scielo.org.co/scielo.php?script=sci_arttext\&pid=S012028122010000100014

Quelal, M. B. (22 de Abril de 2019). Estudio de la comercialización del chocho desamargado (Lupinus mutabilis Sweet) en el Distrito Metropolitano de Quito. (Tesis de Maestría). Universidad Andina Simón Bolívar, Quito. Obtenido de http://repositorio.uasb.edu.ec/bitstream/10644/6650/1/T2877-MAE-QuelalEstudio.pdf

Sanaguano, H., Tigre, A., Bayas-Morejón, F., Ruilova, M.B. (2017). Nutritional value and sensory properties of cookies prepared from flour mixes of carrot (Daucus carota), Lupine (Lupinus perennis) and Barley (Hordeum vulgare). Euro Sci $\mathrm{J} ; 13(9): 378$.

Serna Cock , L., \& Torres León, C. (2015). Potencial agroindustrial de cáscaras de mango (Mangifera indica) variedades Keitt y Tommy Atkins. Acta Agronómica, 64(2), pp.110 - 115. doi:https://doi.org/10.15446/acag.v64n2.43579

Velásquez, S. A. (2012). Estudio de dieta total: Determinación de sodio y potasio en alimentos consumidos por la población de Valdivia. Chile.

Victoria, E. M. (2016). El calcio, esencial para la salud. Nutrición Hospitalaria.

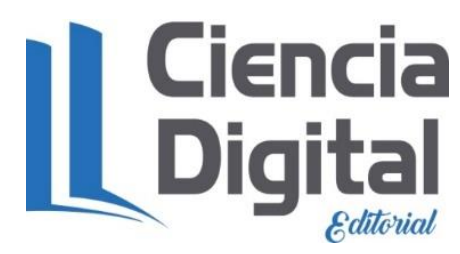




\section{Para citar el artículo indexado}

Rodas Espinoza, S. L., Marcatoma Tixi, J. A., Calderón, A. del R. C., \& Cuadrado, L. Caracterización de la harina de cáscara de chocho (Lupinus mutabilis Sweet) para aplicaciones agroindustriales y medicinales. AlfaPublicaciones, 3(2.2), 6-16. https://doi.org/10.33262/ap.v3i2.2.55

\section{Ciencia \\ LDigital}

El artículo que se publica es de exclusiva responsabilidad de los autores y no necesariamente reflejan el pensamiento de la Revista Alpha Publicaciones.

El artículo queda en propiedad de la revista y, por tanto, su publicación parcial y/o total en otro medio tiene que ser autorizado por el director de la Revista Alpha Publicaciones.
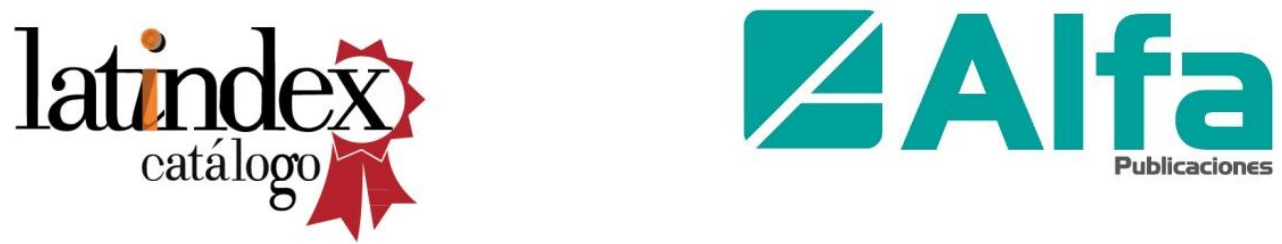